\section{Successful Treatment of Necrolytic Acral Erythema with Ledipasvir and Sofosbuvir}

\section{Shaikh G, Fruchter R, Yagerman S and Franks AG Jr*}

The Ronald O Perelman Department of Dermatology, New York University Langone Medical Center, New York, USA

\begin{abstract}
Importance: Necrolytic Acral Erythema (NAE) is a cutaneous manifestation of hepatitis $C$ virus infection, characterized by painful, unsightly lesions that tremendously diminish quality of life. Currently available treatments are minimally effective or difficult to tolerate. Additionally, interferon alpha, the most effective treatment to date, is contraindicated in patients with autoimmune disease.

Observations: A 58-year-old woman with a history of hepatitis C and dermatomyositis presented for follow up of a 12-year history of a pruritic, painful eruption that affected the feet, ankles, and left calf. Based on clinical and histological findings, a diagnosis of NAE was made. Given her history of dermatomyositis, interferon therapy was contraindicated. Previous treatments with topical steroids and zinc supplementation resulted in minimal improvement. Treatment for hepatitis $\mathrm{C}$ with ledipasvir and sofosbuvir (Harvoni) with ribavirin resulted in substantial improvement of her lesions and resolution of symptomatology two months after treatment initiation.

Conclusion and relevance: Our findings support that treatment with Harvoni is effective for NAE and should be offered to patients with NAE with the goal of sustained viral response. Further study is needed to confirm the long-term benefit of this treatment.
\end{abstract}

\section{Introduction}

Necrolytic Acral Erythema (NAE) is a painful, pruritic condition typically affecting the lower extremities almost universally associated with hepatitis $C$ [1]. To date, no effective treatment with a tolerable side-effect profile has been described [1]. However, new hepatitis $\mathrm{C}$ therapies that lead to sustained virologic response hold promise to treat this important disease.

*Corresponding author: Andrew G Franks Jr, Connective Tissue Diseases Section, The Ronald O Perelman Department of Dermatology, New York University Langone Medical Center, New York, USA, Tel: +1 2122635015 E-mail: Andrew.Franks@nyumc.org

Citation: Shaikh G, Fruchter R, Yagerman S, Franks AG Jr (2016) Successful Treatment of Necrolytic Acral Erythema with Ledipasvir and Sofosbuvir. J Clin Dermatol Ther 3: 016.

Received: November 18, 2015; Accepted: January 01, 2016; Published: January 15, 2016

\section{Case Report}

A 58-year-old woman with a history of hepatitis $\mathrm{C}$ presented to the connective tissue dermatology clinic for follow-up of a 12-year history of a pruritic, painful eruption that affected the feet, ankles, and left calf. Her past medical history was also significant for dermatomyositis in remission for four years. An extensive workup for underlying malignancy was reportedly normal. Medications included IVIG, prednisone, and clobetasol ointment. She had no known allergies.

Physical exam was notable for confluent, well-demarcated, hyperpigmented, lichenified plaques with scalloped borders and overlying, thick, necrotic appearing scale and erythema present on the dorsal aspect of the feet and extending to the medial aspects of the ankles (Figure 1). There was associated $1+$ pitting oedema in the bilateral feet extending to the ankles. Her HCV virus RNA PCR was $8,931,273 \mathrm{IU} / \mathrm{mL}$ (normal $<43 \mathrm{IU} / \mathrm{mL}$ ) and hepatitis $\mathrm{C}$ genotype was 1a. Her zinc level was within normal limits.

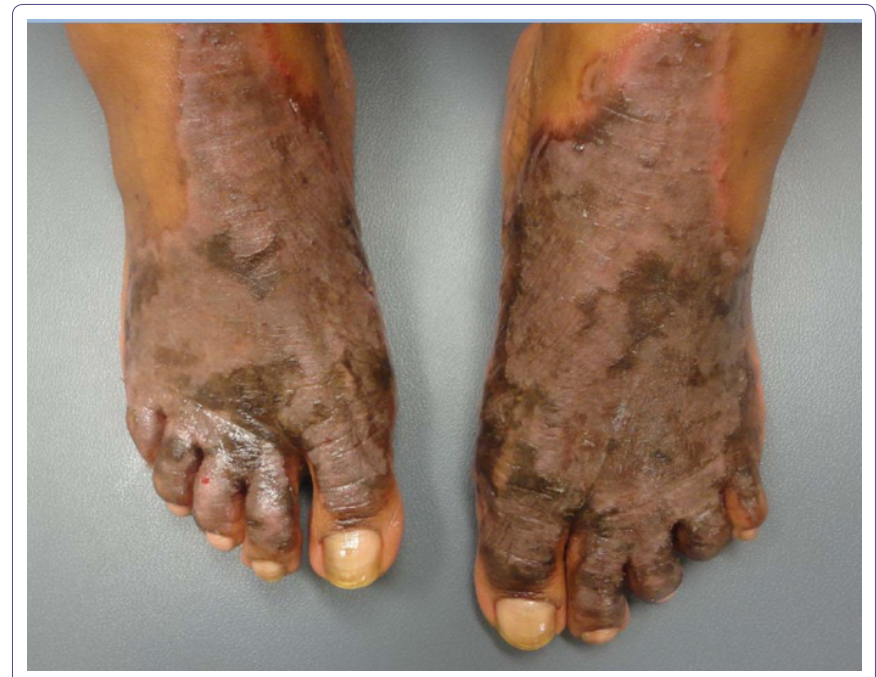

Figure 1: Dorsal feet prior to treatment demonstrating confluent, well-demarcated, hyperpigmented plaques with scalloped borders and overlying, thick, necrotic appearing scale and erythema.

Pathology of a representative lesion revealed slight epidermal hyperplasia, pallor in the superficial portions of the epidermis, and mild spongiosis, as well as confluent parakeratosis with focal collections of neutrophils. Additionally, there were thin-walled blood vessels within some papillae. Based on clinical and histological findings, a diagnosis of Necrolytic Acral Erythema (NAE) was made. Given her history of dermatomyositis, interferon therapy was contraindicated. Previous treatments with topical steroids and zinc supplementation resulted in minimal improvement.

The patient was ultimately treated with ledipasvir $90 \mathrm{mg}$ and sofosbuvir $400 \mathrm{mg}$ (Harvoni) as a combination therapy for her hepatitis $\mathrm{C}$ for 12 weeks. Ribavirin $600 \mathrm{mg}$ was added at six weeks for augmentation [2]. After two months of therapy, the patient reports significantly decreased redness, pain and itching in her bilateral feet and legs. Additionally, she reports resolving lower extremity oedema and is pleased with the improved cosmesis of her feet, which she says 
negatively affected her quality of life. On physical exam, hyperpigmented lichenified stellate plaques with diminished scale were present on the distal feet bilaterally with resolved erythema and oedema (Figure 2). Viral load at the time was below the lower limit of detection.

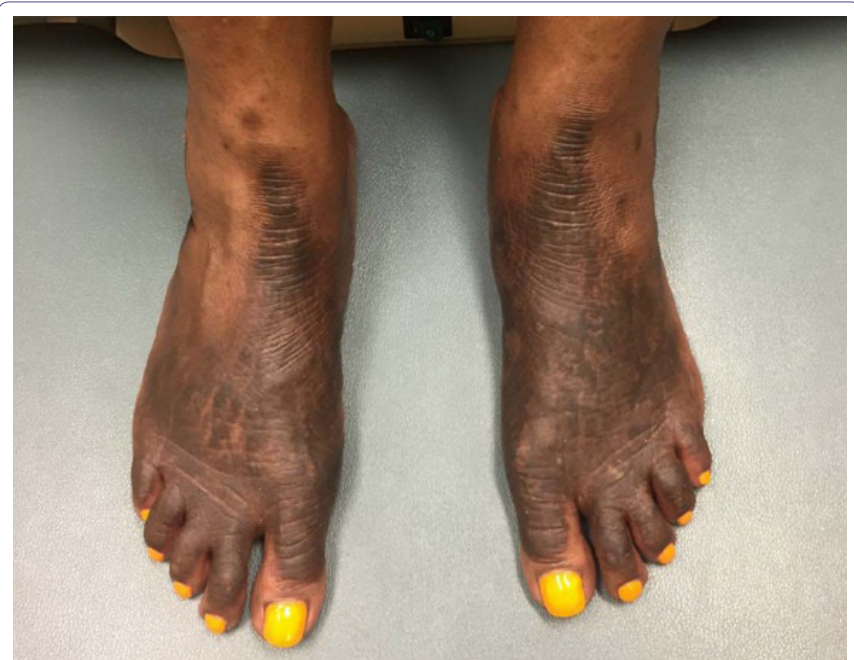

Figure 2: Dorsal Feet after 8 weeks treatment with ledipasvir and sofosbuvir demonstrating hyperpigmented lichenified stellate plaques with diminished scale and resolved erythema and oedema.

\section{Discussion}

NAE is a rare cutaneous manifestation of Hepatitis C Virus (HCV) [1] initially considered to be pathognomonic for the infection however five seronegative cases have been reported [1,3-5]. Initially described among a series of seven Egyptian patients in 1996, there are now over 70 cases of NAE described in the literature likely paralleling the increasing worldwide prevalence of HCV infection [1]. A recent cohort study estimated the prevalence to be $1.7 \%$ among $\mathrm{HCV}$-infected individuals, primarily affecting individuals greater than 40 years old and of African descent [6].

NAE is a part of a wider spectrum of necrolytic erythemas which include necrolytic migratory erythema, acrodermatitis enteropathica, pellagra, and essential fatty acid deficiency [7]. Clinically, early lesions are characterized by erythematous to violaceous papules, blisters, and erosions with associated pruritus and pain [6]. Later in the disease course, lesions progress to well-demarcated, hyperkeratotic plaques with necrotic scale. Secondary lichenification and hyperpigmentation can occur. Unique among the necrolytic erythemas, NAE primarily effects acral sites including the dorsal aspects of the feet and toes, Achilles tendon, malleoli, legs and knees. NAE may involve the hands and nails in a minority of cases. Histologically, acute NAE is characterized by acanthosis, epidermal spongiosis, and a superficial, perivascular infiltrate. In chronic lesions, prominent papillomatosis with parakeratosis, subcorneal pustules, epidermal pallor, and keratinocyte necrosis are seen [6]. This is a relatively non-specific histopathologic pattern that is also seen in other necrolytic erythemas. Thus, correct diagnosis requires a high degree of clinical suspicion and clinical-pathologic correlation.

Zinc deficiency is likely involved in NAE, thought to be related to HCV-induced zinc dysregulation [8]. However, to date, the details of the mechanism by which the virus induces zinc abnormalities has not been elucidated [8]. Zinc supplementation results in clinical improvement in a subset of patients possibly related to zinc's anti-inflammatory, immunostimulatory, antiviral, and antioxidant effects [9]. Additionally, zinc may increase the response to HCV treatment [10]. To date, however, zinc supplementation, which is the mainstay of therapy, results in partial or no response in $87 \%$ of patients [11]

Until recently, interferon alpha with ribavirin, which clears the virus in 40 to 50 percent of cases by an unclear mechanism involving viral suppression, resulted in the most substantial clinical response, however it is poorly tolerated by patients [12]. Further, interferon therapy is contraindicated in autoimmune conditions, as in the patient in this case, due to an association with autoimmune phenomena [12]. Newer, interferon-free combination regimens with ledipasvir and sofosbuvir (Harvoni) have revolutionized the treatment of HCV genotype 1 and result in sustained virologic response, defined as absence of HCV RNA by PCR six months after stopping treatment, in 93 to 100 percent treatment naive patients [2]. Ledipasvir, which inhibits the HCV NS5A protein required for viral replication and sofosbuvir, a prodrug that in the active form inhibits the NS5B RNA-dependent RNA polymerase, are combined into one tablet, which is taken once daily. In general, treatment with Harvoni is extremely well-tolerated.

The pathogenesis of NAE as it relates to $\mathrm{HCV}$ infection is incompletely understood but likely relates to systemic metabolic perturbations including hypoalbuminemia, hypoaminoacidemia, low zinc level, increased glucagon, and liver dysfunction. Severity of the disease may reflect the extent of hepatic involvement, as increasing levels of viremia correlated with worsening NAE [13]. Consistent with this, in the patient described, reduction in her viral load from a peak at $17,603,860$ to below the lower limit of detection was associated with substantial improvement. Thus, therapy should be directed towards diminishing viral load in order to improve NAE.

Patients with NAE describe painful, unsightly lesions that tremendously diminish quality of life. Presented above is the first case of NAE successfully treated with ledipasvir and sofosbuvir. Given the almost universal association with HCV, treatment of the underlying infection with new, interferon-free regimens should be offered to these patients with a goal of sustained virologic response. Due to the lack of effectiveness and/or tolerability of other current treatments, these medications represent a novel solution to this recalcitrant disease. Further research is needed to confirm the long-term benefit of this treatment and whether specific patient populations benefit more or less from this therapy.

\section{Acknowledgement}

This report was funded by the Frances and Benjamin Benenson Foundation.

\section{References}

1. Panda S, Lahiri K (2010) Seronegative necrolytic acral erythema: a distinct clinical subset? Indian J Dermatol 55: 259-261.

2. Afdhal N, Zeuzem S, Kwo P, Chojkier M, Gitlin N, et al. (2014) Ledipasvir and sofosbuvir for untreated HCV genotype 1 infection. N Engl J Med 370: 1889-1898

3. Liu A, Erickson CP, Cockerell CJ, Hsu S (2008) Necrolytic acral erythema: a case not associated with hepatitis C infection. Dermatol Online J 14: 10.

4. Wu YH, Tu ME, Lee CS, Lin YC (2009) Necrolytic acral erythema without hepatitis C infection. J Cutan Pathol 36: 355-358.

5. Nikam BP (2009) Necrolytic acral erythema seronegative for hepatitis $C$ virus-two cases from India treated with oral zinc. Int J Dermatol 48: 1096-1099. 
6. Bentley D, Andea A, Holzer A, Elewski B (2009) Lack of classic histology should not prevent diagnosis of necrolytic acral erythema. J Am Acad Dermatol 60: 504-507.

7. Khanna VJ, Shieh S, Benjamin J, Somach S, Zaim MT, et al. (2000) Necrolytic acral erythema associated with hepatitis C: effective treatment with interferon alfa and zinc. Arch Dermatol 136: 755-757

8. Ko HM, Hernandez-Prera JC, Zhu H, Dikman SH, Sidhu HK, et al. (2012) Morphologic features of extrahepatic manifestations of hepatitis $\mathrm{C}$ virus infection. Clin Dev Immunol 2012: 740138.

9. Abdallah MA, Hull C, Horn TD (2005) Necrolytic acral erythema: a patient from the United States successfully treated with oral zinc. Arch Dermatol 141 85-87.
10. Takagi H, Nagamine T, Abe T, Takayama H, Sato K, et al. (2001) Zinc supplementation enhances the response to interferon therapy in patients with chronic hepatitis C. J Viral Hepat 8: 367-371.

11. Tabibian JH, Gerstenblith MR, Tedford RJ, Junkins-Hopkins JM, Abuav R (2010) Necrolytic acral erythema as a cutaneous marker of hepatitis C: report of two cases and review. Dig Dis Sci 55: 2735-2743.

12. Dietrich LL, Bridges AJ, Albertini MR (2000) Dermatomyositis after interferon alpha treatment. Med Oncol 17: 64-69.

13. El-Ghandour TM, Sakr MA, El-Sebai H, El-Gammal TF, El-Sayed MH (2006) Necrolytic acral erythema in Egyptian patients with hepatitis $C$ virus infection. J Gastroenterol Hepatol 21: 1200-1206. 